\title{
ENERGY METHODS FOR THE CAHN-HILLIARD EQUATION*
}

\author{
BY \\ AMY NOVICK-COHEN ${ }^{1}$ \\ Michigan State University, East Lansing, Michigan
}

Abstract. The Cahn-Hilliard equation, which is important in the context of firstorder phase transition, has frequently been studied in its simplified form,

$$
c_{t}=\Delta[h(c)-K \Delta c],
$$

where $c(x, t)$ is a concentration, $h(c)$ is a nonmonotone chemical potential, and $K$, the coefficient of gradient energy, is a positive constant. In this paper we consider the Cahn-Hilliard equation with nonconstant mobility and gradient energy coefficients,

$$
c_{t}=\nabla \cdot[M(c) \nabla\{h(c)-K(c) \Delta c\}],
$$

where $M(c)$ and $K(c)$ are assumed to be positive. When $K$ is constant, the free energy functional

$$
F(t)=\int_{\Omega}\left\{\int^{c} h(\bar{c}) d \bar{c}+\frac{1}{2} K|\nabla c|^{2}\right\} d x
$$

acts as a Liapounov functional for the Cahn-Hilliard equation. However, when $K$ is nonconstant $F(t)$ no longer acts as a Liapounov functional, and it becomes relevant to examine an alternative energy. In this paper the stability of spatially homogeneous states is studied in terms of the energy

$$
E(t)=\int_{\Omega} \int_{0}^{c-c_{0}} \int_{0}^{\tilde{c}} M^{-1}\left(\bar{c}+c_{0}\right) d \bar{c} d \tilde{c} d x .
$$

The possibility of dependence of $h(c), M(c)$, and $K(c)$ on a spatially uniform temperature is also considered and the physical implications of the location of the limit of monotonic global stability in the average concentration-temperature plane is discussed. In particular, this limit is shown to lie below the critical temperature.

Introduction. The Cahn-Hilliard equation first appeared [2] as a phenomenological model equation for first-order phase transitions with a conserved order parameter. It is possible to derive the Cahn-Hilliard equation if the free energy of the system is assumed to be given by a Gibbs free energy augmented by a gradient energy term [3]

$$
F(t)=\int_{\Omega}\left\{H(c)+\frac{1}{2} K|\nabla c|^{2}\right\} d x .
$$

*Received May 15, 1987.

'This work was supported by the Air Force Office of Scientific Research, Grant \#87-0267. 


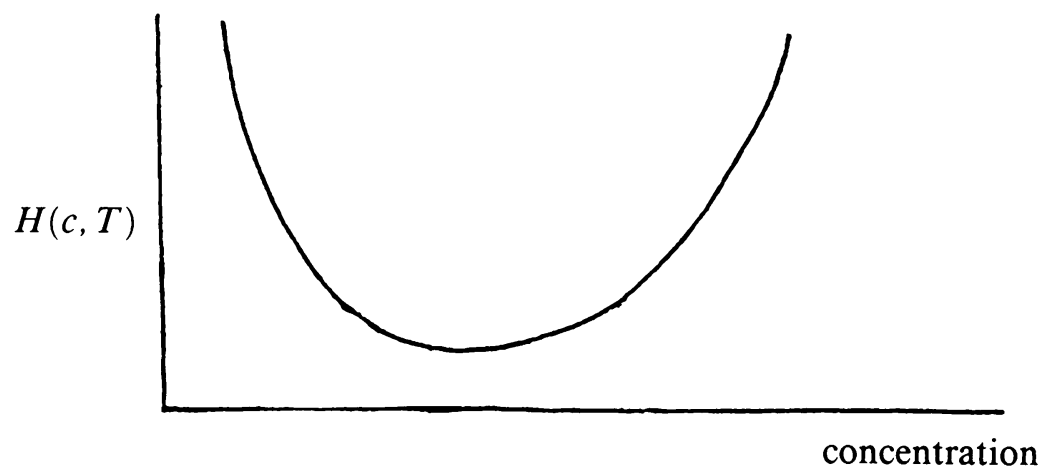

FIG. 1a. The free energy $H(c)$ at some temperature $T_{1}>T_{c}$

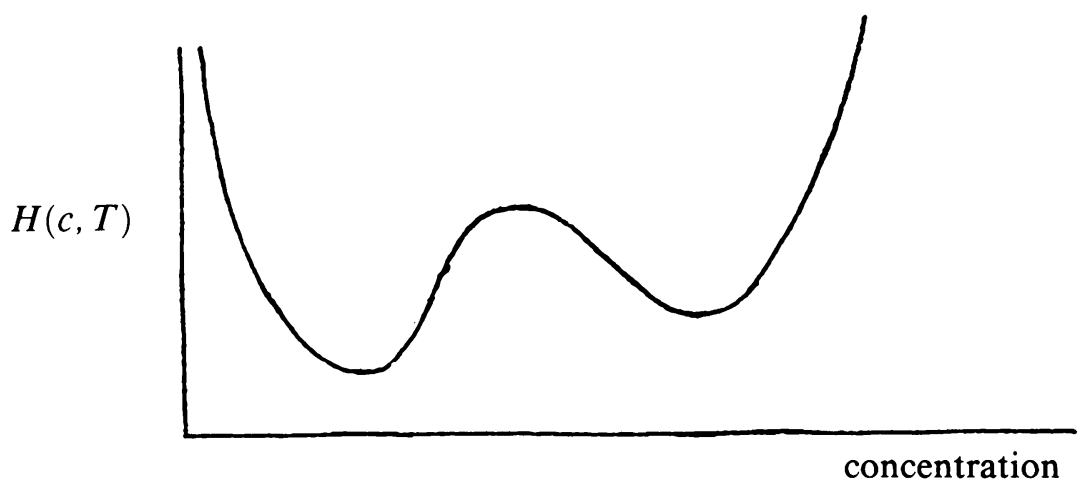

Fig. 1b. The free energy $H(c)$ at some temperature $T_{2}<T_{c}$

Here $H(c)=\int_{0}^{c} h(\bar{c}) d \bar{c}$, where $h(c)$ is the intrinsic chemical potential. The form of $H(c)$ will depend on the temperature. Above some critical temperature $T_{c}, H(c)$ should have one minimum (or well) and below $T_{c}, H(c)$ can be expected to have two minima (or wells). See Figures $1(\mathrm{a}, \mathrm{b})$. The coefficient $K$ is a coefficient of gradient energy which can be assumed to be small and positive. If a generalized chemical potential $\mu$ is defined via

$$
\frac{\delta F}{\delta c}=\mu
$$

and if a linear force-flux relation is assumed, i.e., $J=M \nabla \mu$ where $M>0$ is the mobility, then the Cahn-Hilliard equation follows from mass conservation. Reasonable accompanying boundary conditions on a bound region $\Omega \subset \mathbf{R}^{n}$ are no flux,

$$
\mathbf{n} \cdot J=0 \text { on } \partial \Omega \text {, }
$$


where $\mathbf{n}$ is the normal to the boundary, $\partial \Omega$, together with the natural boundary conditions obtained from the free energy functional,

$$
n \cdot \nabla c=0 \text { on } \partial \Omega .
$$

The inclusion of the gradient energy term arises by allowing the free energy to depend on gradients of concentration as well as on the concentration itself. By assuming the gradients to be small, and by expanding the free energy in the gradients, the gradient term $\frac{1}{2} K|\nabla c|^{2}$ appears as the first nontrivial new contribution in the isotropic system. Thus the Cahn-Hilliard equation can be considered to be valid only if the gradients are reasonably small. If not only are the gradients small but the system as a whole is nearly homogeneous, then it is reasonable to assume that $M$ and $K$ are constants. Here we wish to consider the case where the gradients in the system are small, but where the system as a whole is not nearly homogeneous. One can then either adopt the Cahn-Hilliard equation with nonconstant coefficients,

$$
c_{t}=\nabla \cdot M(c) \nabla[h(c)-K(c) \Delta c],
$$

or return to the free energy function and allow $K$ to be nonconstant. A parallel derivation would then yield

$$
c_{t}=\nabla \cdot M(c) \nabla\left[h(c)-\frac{\partial K}{\partial c}|\nabla c|^{2}-K \Delta c\right] .
$$

The important difference between (1.4) and (1.5) is that while the free energy functional $F$ serves as a Liapounov functional for (1.5), equation (1.4) does not possess a similarly related Liapounov functional. In this paper, equations of the form (1.4) are treated together with the no flux and "natural" boundary conditions, (1.2)-(1.3). On physical grounds Eq. (1.5) may be preferable to Eq. (1.4). However, it is important to consider the properties of Eq. (1.4) in order to obtain a measure of the robustness of the original constant coefficient equation.

In studying the Cahn-Hilliard equation, stability considerations are traditionally connected to the location of the spinodal and the coexistence curves. The spinodal concentrations $\left\{c_{a}, c_{b}\right\}$ are those concentrations such that

$$
h^{\prime}\left(c_{a}\right)=h^{\prime}\left(c_{b}\right)=0
$$

and the coexistence concentrations are those concentrations $\left\{c_{a}^{\prime}, c_{b}^{\prime}\right\}$ such that

$$
h\left(c_{a}^{\prime}\right)=h\left(c_{b}^{\prime}\right)=\left(c_{b}^{\prime}-c_{a}^{\prime}\right)^{-1} \int_{c_{a}^{\prime}}^{c_{b}^{\prime}} h(c) d c .
$$

See Figures $(2 \mathrm{a}, \mathbf{b})$. We denote the interval $\left(c_{a}, c_{b}\right)$ the subspinodal region. If $M$ and $K$ are constant, then the spatially homogeneous state $c(x) \equiv \bar{c}$ is linearly stable if $\bar{c} \notin\left[c_{a}, c_{b}\right]$ and it is linearly unstable otherwise. If $K$ and $M$ are allowed to be concentration dependent, then the stability interpretation of the spinodal and coexistence concentrations is no longer so transparent. We will approach these questions in the next two sections. 

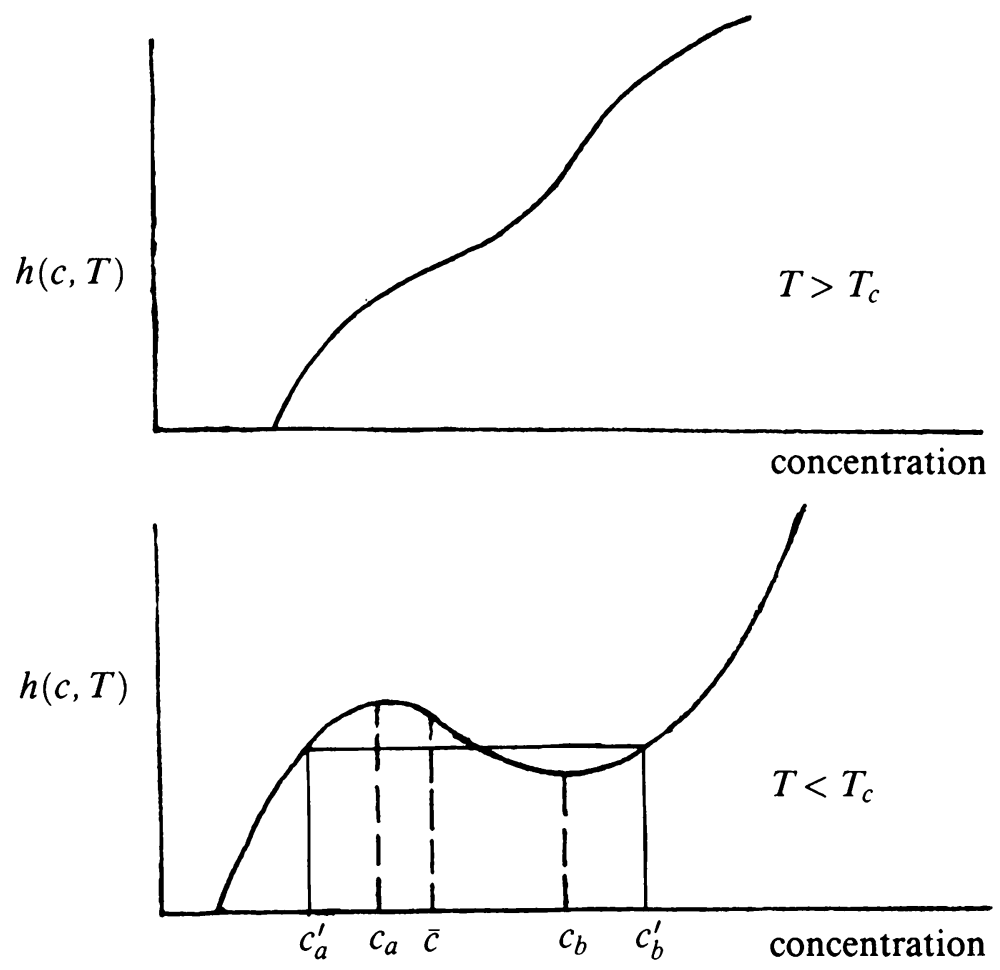

FIG. 2. Typical diagrams for $h=h(c, T)$, for $T>T_{c}$ above and for $T<T_{c}$ below. Here $c_{a}$ and $c_{b}$ denote the spinodal concentrations and $c_{a}^{\prime}$ and $c_{b}^{\prime}$ denote the coexistence concentrations.

2. An energy result. In this section we study the stability of the spatially homogeneous state via the energy

$$
E=\int_{\Omega} \int_{0}^{c-c_{0}} \int_{0}^{\bar{c}} M^{-1}\left(\bar{c}+c_{0}\right) d \bar{c} d \tilde{c} d x .
$$

The physical interpretation of the energy $E$ is unfortunately not transparent. Here we define $c(x, t)$ to be a solution to the initial boundary value problem:

$$
\begin{array}{ll}
c_{t}=\nabla \cdot M(c)[\nabla[h(c)-\Delta K(c) \Delta c]], & x \in \Omega, \\
\mathbf{n} \cdot \nabla c=0, & x \in \partial \Omega, \\
\mathbf{n} \cdot \nabla[h(c)-K(c) \Delta c]=0, & x \in \partial \Omega, \\
c(x, 0)=c_{0}(x) \in C^{4}, &
\end{array}
$$

where $\mathbf{n}$ is the normal to the boundary of the region $\partial \Omega$. Here $\Omega \subset \mathbf{R}^{3}$ is assumed to be a bounded region with a smooth boundary. Furthermore, we assume that $M(c)$, $K(c)$, and $h(c)$ are $C^{1}$, and that there exist constants $M_{0}, K_{0}$, and $K_{1}$ such that for all $c \in R$,

$$
0<M_{0} \leq M(c) \text { and } 0<K_{0} \leq K(c) \leq K_{1} .
$$

The function $h(c)$ is assumed to be monotone increasing or to possess at most one internal minimum, see Figures $1 \mathrm{a}$ and $1 \mathrm{~b}$, and the terminology "spinodal" and "coexistence" concentrations will be understood to be defined by (1.6) and (1.7); in 
particular, $c_{a}$ and $c_{b}$ denote the spinodal concentrations and $h^{\prime}(c) \geq 0$ if $c \in\left[c_{a}, c_{b}\right]$ and $h^{\prime}(c)<0$ otherwise.

A number of existence proofs have been given for Equation $I(a-c)$ for the constant coefficient case (see [7], [8]). A proof of existence for the non-constant coefficient case is not given here, although it should be straightforward; instead we obtain a priori estimates for stability regimes. If the coefficients in $\mathrm{I}(\mathrm{a}-\mathrm{c})$ are constants, then it is well known that the Cahn-Hilliard equation is material conservative [1]. This is also true for $\mathrm{I}(\mathrm{a}-\mathrm{c})$ with variable coefficients, since integrating $\mathrm{I}(\mathrm{a}-\mathrm{c})$ over $\Omega$ yields that

$$
\frac{d}{d t} \int_{\Omega} c(x, t) d x=0
$$

or equivalently, that

$$
\frac{1}{\operatorname{vol}(\Omega)} \int_{\Omega} c(x, t) d x=\frac{1}{\operatorname{vol}(\Omega)} \int_{\Omega} c_{0}(x) d x=\bar{c}_{0}
$$

for all $t>0$.

Let $\chi(c)$ be the characteristic function of the subspinodal region (see Figures $2(\mathrm{a}, \mathrm{b}))$ :

$$
\chi(c)= \begin{cases}1 & c \in\left[c_{a}, c_{b}\right] \\ 0 & c \notin\left[c_{a}, c_{b}\right]\end{cases}
$$

Integrating by parts and employing the boundary conditions $I(b-c)$, we find that

$$
\begin{aligned}
\frac{d}{d t} E(t) & =-\int_{\Omega} h^{\prime}(c)|\nabla c|^{2} d x-\int_{\Omega} K(c)(\Delta c)^{2} d x \\
& =-\int_{\Omega} h^{\prime}(c)|\nabla c|^{2} \chi(c) d x-\int_{\Omega} h^{\prime}(c)|\nabla c|^{2}(1-\chi(c)) d x \\
& -\int_{\Omega} K(c)(\Delta c)^{2} d x .
\end{aligned}
$$

Let us define

$$
\begin{gathered}
P=-\int_{\Omega} h^{\prime}(c)|\nabla c|^{2} \chi(c) d x, \\
D=\int_{\Omega} h^{\prime}(c)|\nabla c|^{2}(1-\chi(c)) d x+\int_{\Omega} K(c)(\Delta c)^{2} d x .
\end{gathered}
$$

Recalling that $h^{\prime}(c) \geq 0$ if $c \notin\left[c_{a}, c_{b}\right]$, the subspinodal region, we see that $D>0$ if $c \not \equiv$ constant and thus

$$
\frac{d}{d t} E(t)=P-D=-D[1-P / D]
$$

We define a class of admissible functions,

$$
A\left(\bar{c}_{0}\right)=\operatorname{cl}\left\{c(x) \in C^{4} \mid c(x)\right. \text { satisfies the boundary conditions }
$$

(I.b) and (I.c) and $c(x)$ satisfies the conservation

$$
\text { of material constraint (2.1)\} . }
$$

If $c(x) \in A\left(\bar{c}_{0}\right)$, then $c(x)-\bar{c}_{0}$ is a material conservative perturbation about the uniform state $c(x) \equiv \bar{c}_{0}$. 
Claim 1. Let $Q=\max _{c \in[0,1]}\left(-h^{\prime}(c)\right)$. There exists a positive constant $\Lambda(\Omega)$ such that for all admissible functions, $P / D \leq R_{1}$ where $R_{1}=Q \Lambda(\Omega) / K_{0}$ if $\left[c_{a}, c_{b}\right] \neq \varnothing$ and $R_{1}=0$ otherwise. Here $K_{0}$ is a positive lower bound on $K(c)$.

Claim 2. For all admissible functions, $E \leq R_{2} D$ where $R_{2}=\Lambda^{2}(\Omega) /\left(2 K_{0} M_{0}\right)$. Here $\Lambda(\Omega)$ is the same positive constant which appears in Claim 1 and $M_{0}$ is a positive lower bound on $M(c)$.

From Claim 1, it follows that $\nu(t)=\sup _{c(x, 0) \in A}\{P(t) / D(t)\}<\infty$.

Theorem 1. If $\nu(t)<1$ for all $t \in[0, T)$, then for all $t \in[0, T]$

$$
E(t) \leq E(0) \exp \left\{-R_{2}^{-1} \int_{0}^{t}\left(1-\nu\left(t^{\prime}\right)\right) d t^{\prime}\right\}
$$

where $R_{2}$ is defined in Claim 2. If all perturbations about a particular state $c(x)$ decay monotonically, then we say that that state is monotonically and globally stable. In particular, if $\tilde{\nu}=\sup _{t>0} \nu(t)<1$ for all $t$, then $c \equiv c_{0}$ is monotonically and globally stable. If $\nu(0) \geq 1$, then there exist initial conditions for which $d E(0) / d t \geq 0$.

Proof. The proof of Theorem 1 follows from Claims 1 and 2 together with standard arguments. See, for example, [5], $\S 4$.

We now proceed with the proofs of Claims 1 and 2. Let us first prove that for $c(x) \in A$

$$
\int_{\Omega}|\nabla c|^{2} d x \leq \Lambda(\Omega) \int_{\Omega}(\Delta c)^{2} d x
$$

Integrating by parts,

$$
\begin{aligned}
\int_{\Omega}|\nabla c|^{2} d x & =-\int_{\Omega}\left(c-c_{0}\right) \Delta c d x \leq \int_{\Omega}\left|c-c_{0}\right||\Delta c| d x \\
& \leq\left[\int_{\Omega}\left(c-c_{0}\right)^{2} d x\right]^{1 / 2}\left[\int_{\Omega}(\Delta c)^{2} d x\right]^{1 / 2} .
\end{aligned}
$$

By Poincarés inequality for functions with zero mean,

$$
\int_{\Omega}|\nabla c|^{2} d x \leq \Lambda^{1 / 2}(\Omega)\left[\int_{\Omega}|\nabla c|^{2} d x\right]^{1 / 2}\left[\int_{\Omega}(\Delta c)^{2} d x\right]^{1 / 2},
$$

from which $(*)$ follows.

Proof of Claim 1. The proof proceeds by bounding $-\int_{\Omega} h^{\prime}(c)|\nabla c|^{2} \chi(c) d x$ in terms of $\int_{\Omega} K(c)(\Delta c)^{2} d x$. If range $c(x, t) \cap\left[c_{a}, c_{b}\right]=\varnothing$, then the result is trivially true. Otherwise $0<-\min _{c \in[0,1]} h^{\prime}(c) \chi(c) \leq Q$ and from $(*)$ it follows that

$$
\begin{aligned}
-\int_{\Omega} h^{\prime}(c)|\nabla c|^{2} \chi(c) d x & \leq Q \int_{\Omega}|\nabla c|^{2} d x \leq Q \Lambda(\Omega) \int_{\Omega}(\Delta c)^{2} d x \\
& \leq \frac{Q}{K_{0}} \Lambda(\Omega) \int_{\Omega} K(c)(\Delta c)^{2} d x .
\end{aligned}
$$

Thus $P \leq R_{1} D$ where $R_{1}=Q \Lambda(\Omega) / K_{0}$. 
Proof of Claim 2. Applying first Poincarés inequality for functions with zero mean and then using again the inequality $(*)$, we obtain

$$
\begin{aligned}
E(c(x, t)) & =\int_{\Omega} \int^{c-c_{0}} \int^{\tilde{c}} M^{-1}\left(\bar{c}+c_{0}\right) d \bar{c} d \tilde{c} d x \\
& \leq \frac{1}{M_{0}} \frac{1}{2} \int_{\Omega}\left(c-c_{0}\right)^{2} d x \leq \frac{\Lambda(\Omega)}{2 M_{0}} \int_{\Omega}|\nabla c|^{2} d x \\
& \leq \frac{\Lambda^{2}(\Omega)}{2 M_{0}} \int_{\Omega}(\Delta c)^{2} d x \leq \frac{\Lambda^{2}(\Omega)}{2 K_{0} M_{0}} \int_{\Omega} K(c)(\Delta c)^{2} d x .
\end{aligned}
$$

When $K(c)=$ constant, it is possible to compare the behavior of $E(t)$ and $F(t)$. The following result shows that even if $E(t)$ does not decrease monotonically, its behavior is controlled by the behavior of $F(t)$.

Theorem 2. There exists a constant $C=C(c(x, 0), K)$ such that $\left|\frac{d E}{d t}\right| \leq \frac{C}{K}\left(-\frac{d F}{d t}\right)^{1 / 2}$.

Proof. Integrating by parts and using Cauchy-Schwarz, one obtains

$$
\begin{aligned}
\left|\frac{d E}{d t}\right| & =\left|\int_{\Omega} \int^{c-c_{0}} M^{-1}\left(\bar{c}+c_{0}\right) d \bar{c} \nabla \cdot\left[M \nabla\left\{h(c)-K \nabla^{2} c\right\}\right] d x\right| \\
& =\left|\int_{\Omega} \nabla c \cdot\left[\nabla\left\{h(c)-K \nabla^{2} c\right\} d x\right]\right| \\
& \leq\left[\int_{\Omega}|\nabla c|^{2} d x\right]^{1 / 2}\left[\int_{\Omega}\left|\nabla\left\{h(c)-K \nabla^{2} c\right\}\right|^{2} d x\right]^{1 / 2}<\left[\int_{\Omega}|\nabla c|^{2} d x\right]^{1 / 2}\left(-\frac{d F}{d t}\right)^{1 / 2} .
\end{aligned}
$$

Furthermore, since $\frac{d F}{d t} \leq 0$,

$$
F(c(x, t))=\int_{\Omega}\left\{\int^{c} h(\bar{c}) d c+K|\nabla c|^{2}\right\} d x \leq F(c(x, 0)) .
$$

Hence, since $\int^{c} h(\bar{c}) d \bar{c}$ is of quartic form with two minima,

$$
\begin{aligned}
\int_{\Omega} K|\nabla c|^{2} d x & \leq F(c(x, 0))-\int_{\Omega} \int^{c} h(\bar{c}) d \bar{c} d x \leq F(c(x, 0))-\min _{c} \int^{c} h(\bar{c}) d \bar{c} \cdot|\Omega| \\
& =C(c(x, 0), K) .
\end{aligned}
$$

3. Physical implications. In the previous sections we have allowed $M, K$, and $h$ to be concentration dependent. It is well known that $M, K$, and $h$ will also be expected to vary with temperature. In this section, we take the temperature to be an additional variable, but we assume that in any particular system, the temperature is fixed and independent of space and time. We examine the dependence of monotonic global stability on the variables $T$ and $c_{0}$, where $T$ is the ambient temperature and where $c_{0}=\frac{1}{\Omega} \int_{\Omega} c_{0}(x) d x$.

Typically (e.g., for dilute binary solutions) the interval $\left[c_{a}^{\prime}, c_{b}^{\prime}\right]$ shrinks as the temperature is raised and $Q=-\min h^{\prime}(c, T)$ decreases, until at some critical temperature $T_{c}<\infty, c_{a}^{\prime}=c_{b}^{\prime}$ and $Q=0$. Likewise the spinodal interval $\left[c_{a}, c_{b}\right]$ may be expected to decrease. Let us define the region of monotonic global stability to be the set $S$,

$$
S=\left\{\left(c_{0}, T\right) \mid \tilde{\nu}\left(c_{0}, T\right)<1\right\}
$$

Later we shall see that $T_{s}\left(c_{0}\right)=\left(\inf T \mid\left(c_{0}, T\right) \in S\right)$ is a well-defined function of $c_{0}$ which we may call the limit of monotonic global stability in the $\left(c_{0}, T\right)$-plane. 


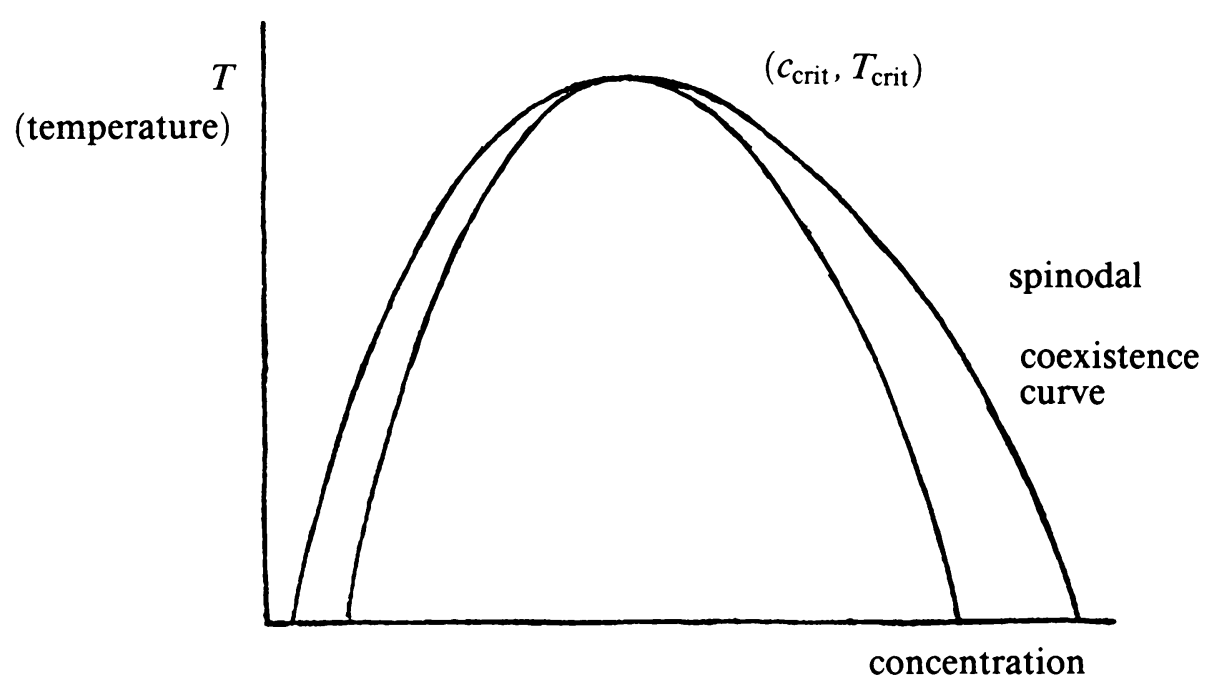

Fig. 3. A typical plot of the spinodal and the coexistence concentrations in the temperature-concentration plane. According to Result $3.1,\left(c_{0}, T\right) \in S$ if $T>T_{c}$.

Result 3.1. If $T>T_{c}$, then $\left(c_{0}, T\right) \in S$. See Fig. 3 .

Proof. If $T>T_{c}$, then by hypothesis the interval $\left[c_{a}, c_{b}\right]$ is empty. Hence $P / D=0$ for all admissible functions.

In accordance with the explanation of the influence of temperature on the free energy functional given at the beginning of this section, we assume that if $T_{2}>T_{1}$, then $h^{\prime}\left(c, T_{2}\right)>h^{\prime}\left(c, T_{1}\right)$. We then obtain

Result 3.2. If $\left(c_{0}, T_{1}\right) \in S$ and if $T_{2}>T_{1}$, then $\left(c_{0}, T_{2}\right) \in S$.

Proof. Suppose that $\left(c_{0}, T_{2}\right) \notin S$. Then there exists an admissible function for which $P / D>1$. By using the inequality $h^{\prime}\left(c, T_{1}\right)>h^{\prime}\left(c, T_{2}\right)$, it follows that at $\left(c_{0}, T_{1}\right)$, for the same admissible function, $P / D>1$. This is a contradiction to the assumption that $\left(c_{0}, T_{1}\right) \in S$.

Result 3.2 implies that $T_{s}\left(c_{0}\right)$ is a well-defined function of $c_{0}$ which may assume the value $-\infty$. It is difficult to obtain information about the exact shape of $T_{s}\left(c_{0}\right)$.

It is also possible to obtain the following characterization of $S$ in terms of the steady state solutions of the Cahn-Hilliard equation.

Result 3.3. If there exist nontrivial steady state solutions of the original equation (1.4) corresponding to the parameter values $\left(c_{0}, T\right)$, then $\left(c_{0}, T\right) \notin S$.

Proof. Any nontrivial steady state solution may be used as an initial condition for which $P / D=1$.

In particular for constant $K$, since extremals of the free energy functional are steady state solutions of (1.4), we have

Result 3.3a. If $K$ is constant, then $\left(c_{0}, T\right) \notin S$ if there exist admissible functions which are extremals of the free energy functional corresponding to the parameter values $\left(c_{0}, T\right)$. 
Some notion of where nontrivial extrema are located may be obtained by invoking the results of Carr, Gurtin, and Slemrod [6].

1) In one dimension, when $K$ is positive and sufficiently small and $c_{0} \in\left(c_{a}^{\prime}, c_{b}^{\prime}\right)$, then the free energy has a unique nontrivial global minimizer.

2) In one dimension, when $K$ is positive and sufficiently small and $c_{0} \in\left(c_{a}^{\prime}, c_{b}^{\prime}\right)$, then the free energy has periodic extrema for all $N, 0 \leq N \leq N_{0}$.

In these results, the smallness of $K$ necessarily is dictated by the proximity of $c_{0}$ to the coexistence concentrations $c_{a}^{\prime}$ and $c_{b}^{\prime}$. Thus nontrivial global monotonic minimizers and periodic extrema exist throughout a region under the coexistence curve bounded away from the coexistence concentrations. Thus most of the area under the coexistence curve lies outside $S$. However, the top of the coexistence curve located at $\left(\bar{c}, T_{c}\right)$ for some $\bar{c}$ lies within $S$. This is true both for constant and nonconstant $K$ and is not limited to one dimension.

Result 3.4. $T_{s}\left(c_{0}\right)<T_{c}$ for all $c_{0}$.

Proof. As in the proof of Claim 1,

$$
\begin{aligned}
-\int_{\Omega} h^{\prime}(c)|\nabla c|^{2} d x & \leq-\min _{c} h^{\prime}(c) \int_{\Omega}|\nabla c|^{2} d x \\
& \leq-\min _{c} h^{\prime}(c) \frac{1}{K_{0} \Lambda(\Omega)} \int_{\Omega} K(c)\left(\nabla^{2} c\right)^{2} d x .
\end{aligned}
$$

Since by hypothesis on the temperature dependence of the coexistence curve, $\min _{c} h^{\prime}(c) \uparrow 0$ and $T \uparrow T_{c}$, it follows that for $T_{c}-T$ sufficiently small,

$$
\begin{aligned}
\frac{d E(t)}{d t} & \leq \int_{\Omega}\left[-h^{\prime}(c)|\nabla c|^{2}-K(c)\left(\nabla^{2} c\right)^{2}\right] d x \\
& \leq-\left[\min _{c} h^{\prime}(c) \frac{1}{K_{0} \Lambda(\Omega)}+1\right] \int_{\Omega} K(c)\left(\nabla^{2} c\right)^{2} d x \leq 0 .
\end{aligned}
$$

Hence all perturbations decay monotonically.

4. Discussion. Traditionally two stability limits have been studied in the context of the Cahn-Hilliard equation-the spinodal curve and the coexistence curve. Both limits have only limiting dynamic relevance. The spinodal curve is the locus of the region of linear stability if the mobility is constant. The coexistence curve is the region in which the free energy function has nonuniform extrema for $K=0$. Even if $K$ is constant, but $M$ is concentration-dependent, the spinodal curve no longer has the meaning of linear stability and the region in which (for $K$ constant) there exist nonhomogeneous extremals lies inside the traditional coexistence curve.

In the general case of nonconstant $K$ and $M$ it would be relevant to study the true limit of linear stability and the region in which there exist nonhomogeneous stationary solutions. A means of approaching stability questions in the general case is facilitated through the energy, which has been defined here as

$$
E=\int_{\Omega} \int_{0}^{c-c_{0}} \int_{0}^{\tilde{c}} M^{-1}\left(\bar{c}+c_{0}\right) d \bar{c} d \tilde{c} d x
$$


This energy allows a region of monotonic global stability of the homogeneous state, $S=S\left(c_{0}, T\right)$, to be defined. Under certain reasonable hypotheses on the temperature dependence of the free energy, $T_{s}=T_{s}\left(c_{0}\right)$ is a well-defined function in the $\left(c_{0}, T\right)$ plane. The curve $T_{s}\left(c_{0}\right)$ lies outside the region in which nontrivial stationary solutions to (1.5) exist. In particular, this implies that for $K$ constant, $T_{s}\left(c_{0}\right)$ lies outside the region in which nontrivial extremals of the free energy functional exist, which corresponds roughly to a region just inside the coexistence curve. Both for constant and nonconstant $K$ we have that $\max _{c_{0}} T_{s}\left(c_{0}\right)<T_{c}$. Lastly, energy result 2 shows that for constant $K$, the growth of the energy $E$ is controlled by the growth of $F(t)$.

While $E$ may not be the optimal choice, it has been shown here to be a useful tool for studying stability in cases where the free energy functional with gradient energy terms is no longer applicable.

Acknowledgments. The author wishes to express her thanks to Hans Weinberger and to Bob Kohn for helpful questions and comments.

\section{REFERENCES}

[1] A. Novick-Cohen and L. A. Segel, Nonlinear aspects of the Cahn-Hilliard equation, Physica 10D, 277-298 (1984)

[2] J. W. Cahn and J. E. Hilliard, Free energy of a nonuniform system. I. Interfacial free energy, J. Chem. Phys. 28, 258-267 (1958)

[3] J. D. van der Waals, The thermodynamimc theory of capillarity under the hypothesis of a continuous variation in density (in Dutch), Verhandel. Konik. Akad. Weten. Amsterdam 1, No. 8 (1893)

[4] G. I. Sivashinsky, On cellular instability in the solidification of a dilute binary alloy, Physica 8D, 243-248 (1983)

[5] D. D. Joseph, Stability of Fluid Motions I., Springer, Berlin (1976)

[6] J. Carr, M. E. Gurtin, and M. Slemrod, Structural phase transitions on a finite interval, Arch. Rat. Mech. Anal. 87, 317-351 (1984)

[7] C. M. Elliott and S. Zheng, On the Cahn-Hilliard equation, Arch. Rat. Mech. Anal. 96, 339-357 (1986)

[8] W. von Wahl, On the Cahn-Hilliard equation, Delft Progr. Rep. 10, 291-301 (1985) 